\title{
An Integrated Analysis of Liver Safety Data from Orlistat Clinical Trials
}

\author{
Marc Morris Peter Lane Kwan Lee Daniel Parks \\ GlaxoSmithKline Research and Development, Collegeville, PA, USA
}

\author{
Key Words \\ Epidemiology • Obesity management • Pharmacological therapy • Drug toxicity • \\ Meta-analysis
}

\begin{abstract}
Objective: Orlistat is an oral gastrointestinal lipase inhibitor and is indicated for treatment of obesity in combination with a hypocaloric diet. Post-marketing reports of adverse reactions revealed hints for possible drug-induced liver injury which has prompted changes to the product information. Orlistat's development program, involving over 30,000 patients, did not indicate a hepatic safety issue. Methods: We analyzed liver function test data from randomized clinical trials of orlistat, using i) meta-analysis of published study safety data, ii) time-to-event analysis for individual patients, and iii) a novel and more sensitive method derived from the US Food and Drug Administration's (FDA) Evaluation of Drug-Induced Serious Hepatotoxicity (eDISH) technique. Over 10,000 subjects were included. Results: The combined odds ratio from a simple summary-level fixed-effects meta-analysis of treatmentemergent abnormalities in serum alanine aminotransferase (ALT) (defined as greater than the upper level of normal for 2 successive measurements) was 1.09 (95\% CI 0.93-1.28), and in total bilirubin 1.24 (95\% CI 1.03-1.49). Part of the small apparent effect was due to longer exposure to orlistat than to placebo, on average. A patient-level display, adjusting for regression towards the mean, and Kaplan-Meier analysis of changes in ALT and bilirubin, taking account of different exposure, showed no significant difference between orlistat and placebo. This shows that there is no signal for hepatic damage in clinical studies of orlistat. Conclusion: While idiosyncratic liver injury following exposure to orlistat cannot be excluded, it is likely to be extremely rare.




\section{Introduction}

Drug-induced liver injury (DILI) is the most common cause of post-marketing regulatory activities taken in response to safety signals [1]. Based on post-marketing adverse event reports, the US Food and Drug Administration (FDA) identified severe liver injury as a potential safety concern with orlistat use. Between 1999 and 2008, 32 reports of serious liver injury, including 6 cases of liver failure, were submitted to FDA's Adverse Event Reporting System. Scientific review of these cases identified 13 cases of severe liver injury possibly related to orlistat. Although a cause-and-effect relationship was not established, the FDA has added a warning regarding possible severe liver injury to the product information for orlistat [2].

Orlistat is a reversible long-acting inhibitor of gastrointestinal lipases, which exerts its therapeutic activity in the lumen of the stomach and small intestine. The inhibition of triglyceride digestion reduces lipid absorption, with a subsequent positive effect on the weight control. Orlistat is indicated for the treatment of obesity in combination with a hypocaloric diet. It is available on prescription (Xenical ${ }^{\circledR} 120$ mg capsules; Roche , Basel, Switzerland) or over-the-counter (Alli ${ }^{\circledR} 60 \mathrm{mg}$ capsules; Roche). Systemic absorption of orlistat is almost undetectable [3], and a clinical development program involving over 30,000 patients did not detect a hepatic safety signal. Nonetheless, aberrant metabolic or immune responses in a small number of individuals may result in idiosyncratic DILI that is only detectable with post-marketing drug surveillance [4]. However, it has also been reported that up to $20 \%$ of acute liver failure cases have no identifiable etiology [5].

Evaluating hepatic effects associated with orlistat is complicated by the fact that obesity is a common cause of non-alcoholic steatohepatitis (NASH), a condition that is usually asymptomatic but can become clinically significant. Furthermore, obese patients, and especially obese diabetics, are at increased risk of acute liver failure [6, 7]. A recent 36-week study showed that weight loss improved fatty liver, related to obesity, though any effects of orlistat were not significant in this 50-patient study [8]. The presence of this source of confounding, in the context of post-marketing reports of serious events, prompted us to conduct a meta-analysis of liver function test (LFT) data from randomized controlled trials of orlistat, which we report in this article.

\section{Material and Methods}

Source of Clinical Trial Data

Roche searched records of the clinical development of orlistat during the 1990s and subsequent postmarketing studies. We restricted attention to studies lasting 4 weeks or longer, because idiosyncratic DILI typically develops up to 2 months after starting treatment [9]. Data were found from eight studies in phase 2 and 3, and a further 8 studies in phase 4. A search of the GlaxoSmithKline (GSK) Clinical Study Register found 1 recent study (3600586). In the studies sponsored by Roche and GSK, approximately $90 \%$ of the subjects were White/Caucasian and 9\% were Black, Oriental or Hispanic.

Literature Search and Data Integration

We conducted a literature search using Embase.com (which includes Medline). The search terms were 'orlistat', 'Xenical' or 'Alli', with 'placebo' and 'clinical trial', restricted to papers in English, with an abstract, and about research on humans. This produced 469 papers. Reading the abstracts yielded 67 reports of studies with duration 4 weeks or longer plus 16 meta-analyses, giving a further 17 reports of studies. (The meta-analyses were all of efficacy or attrition.) Investigating these reports further, we found:

- 10 repeats (i.e. papers with further analysis of studies already published);

- 25 Roche studies, including 10 of the 16 listed in;

- 17 studies with no placebo arm or of duration shorter than 4 weeks; 
- 8 reports that were not available (e.g. abstracts from conference presentations);

- 5 reports with no contact e-mail address or with one that was no longer valid.

We contacted the authors of the remaining 19 studies, and were provided with data from 5 of them (see 'Acknowledgements'). Four further authors replied that the data we needed were not available, and 10 authors did not reply to the enquiry or a follow-up.

We did not assess individual studies for bias such as allocation concealment, because the response variables to be analyzed are laboratory measurements.

\section{Population-Based Analysis of Abnormal ALT and Bilirubin Measurements}

We focused on serum ALT and total bilirubin because there is some agreement that these are useful diagnostic tests of potential DILI. We analyzed only the first year's data from long studies, as the onset of DILI is very likely to eventuate within this period, with spurious changes in liver function tests most likely thereafter. In addition, we used data from only the first period of cross-over studies to avoid the possibility of carry-over and time effects.

We analyzed the proportions of patients who exhibited any treatment-emergent abnormally high values, for both LFTs separately, where 'abnormally high' was taken as greater than the upper limit of normal measurements (ULN) established in each study. To filter out most of the expected chance high fluctuations, we also analyzed a second variable: the proportion of patients with two consecutive abnormally high values, at least 2 weeks apart. Missing data were rare. Counts of subjects randomized to each treatment arm were not adjusted for missing values, unless a subject had no post-treatment measurement of the LFT variable. Missing values were taken as lying within the normal range, except that a moderately pessimistic strategy was used for the second variable: patients with an abnormal value at one visit and a missing value at the next were considered to have two consecutive abnormal values. This analysis takes no account of potential effects caused by differential drop-out rates between the two treatments; this issue is discussed later.

Our protocol, published in the GSK Clinical Study Register in April 2010, stated the primary analysis to be a comparison of orlistat $120 \mathrm{mg}$ with placebo for the two variables mentioned above, for both ALT and bilirubin. The same analyses comparing orlistat $60 \mathrm{mg}$ with placebo were considered secondary. We made no adjustment for multiplicity of significance tests.

We analyzed the summary statistics from each study adjusting for a fixed study effect, using logistic regression with no adjustment for zero counts [10]. We also carried out a random-effects analysis (DerSimonian-Laird) to check sensitivity of the results to the assumption of a fixed effect. We assessed heterogeneity visually in forest plots, including $\mathrm{I}^{2}$ statistics.

\section{Individual Patient-Based Analysis of ALT and Bilirubin Measurements}

The assessment of potential DILI has emerged as a challenging task for the pharmaceutical industry and for drug regulatory agencies because the incidence of a DILI case is rare, but massive volumes of data must be analyzed to have any chance of detecting events. Based on the ideas of the late Hy Zimmerman [11], FDA scientists proposed a graphical method known as eDISH (Evaluation of Drug Induced Serious Hepatotoxicity) [12], which is currently the method used for regulatory reviews of new drug applications. eDISH examines a combination of both ALT and bilirubin values so that subjects of concern may be detected. The method begins by plotting the peak bilirubin (as a multiple of ULN) against the peak ALT (we note that the peak bilirubin and peak ALT for each subject may not occur simultaneously). Two reference lines, $2 \times$ ULN for bilirubin and $3 \times$ ULN for ALT, are then drawn to divide the plane into four quadrants. The normal cases occur in the lower left quadrant. The critical region is the upper right quadrant, known as the Hy's Law quadrant, which includes cases suspected to be serious DILI. The upper left quadrant is referred to as the cholestasis range (high bilirubin but normal ALT). The lower right quadrant is referred as Temple's Corollary range (high ALT but normal bilirubin), which includes cases suspected to be DILI, but with a signal of lower magnitude than for Hy's law. The rationale for Hy's Law is that retrospective analyses of LFT data from clinical trials of drugs subsequently found to be hepatotoxic has indicated that most of these drugs could have been identified if such criteria were applied. However, the sensitivity and specificity of this criterion to identify hepatotoxic drugs has not been formally quantified.

A weakness of eDISH is that, since only peak values are considered, any information about the baseline (BL) is completely disregarded. This information can actually be very important, since consideration of BL values allows post-treatment changes to be measured directly and since BL values them- 
selves may be predictive of the amount of changes that cannot be detected by reliance on laboratory reference ranges which may be too insensitive to detect low-amplitude hepatotoxicity [13]. In this analysis, we have therefore applied eDISH to the BL-scaled measurement for each subject rather than to the unscaled measurement, and will call the method 'modified eDISH' (mDISH). In the absence of further knowledge, we have used similar reference lines in mDISH as in eDISH $(2 \times$ BL for bilirubin and $3 \times \mathrm{BL}$ for ALT) and divided the plane into four quadrants. In $\mathrm{mDISH}$, the upper right quadrant is called Type I (defined using $2 \times$ BL for bilirubin and $3 \times$ BL for ALT). The upper left and lower right quadrants are denoted Type II ( $2 \times$ BL of bilirubin) and Type III ( $3 \times$ BL of ALT), respectively. The lower left quadrant contains normal subjects. mDISH can allow us to examine cases more closely than eDISH, which identifies fewer cases of potential DILI than does mDISH. The mDISH method is also subject to a statistical phenomenon called 'regression toward the mean' that can make natural variation in repeated data seem like a real change. It happens when subjects are selected, wholly or partly, on the basis of a cut-off point of the baseline measurements, because there is then a tendency for subsequent measurements to be closer to the population mean (excluding the restriction of the cut-off). To counter this effect, we adjust values for 'regression toward the mean' when we use the change from baseline using the formula by Chuang-Stein [14]; our mDISH display is on the log scale, so we applied the additive change-from-baseline formula to the logged variables.

As in the binary meta-analysis, no account is taken in the eDISH or mDISH method of potential effects caused by differential drop-out rates between the two treatments. To handle this, we used a Kaplan-Meier (K-M) survival analysis of events defined by the three quadrants of the mDISH display [15]. The method can be used to measure the fraction of patients with safety signals for a given time after treatment. An important characteristic of the K-M analysis is that the method can take into account 'censoring' in the event data. Censoring happens when a particular subject is lost before the final outcome is observed or a subject does not have a safety event until the end of study. The log-rank test provides a p-value for the K-M method. Further research may be necessary to find more accurate reference lines based on historical data for mDISH, but it should be emphasized that, regardless of the validity of the reference lines, comparison of drugs using K-M analysis is still statistically valid.

\section{Results}

\section{Population-Based Analysis of Abnormal ALT and Total Bilirubin Measurements}

Figure 1 summarizes the proportions of patients with two successive abnormal ALT measurements, comparing orlistat patients (60 and $120 \mathrm{mg}$ combined) to placebo. It shows a conventional forest plot enhanced with a dot plot comparing the actual proportions (numbers are also given against the right-hand axis), the $\mathrm{I}^{2}$ statistic (in the bottom righthand corner), and adjusted mean proportions across the set of studies (to the right of the combined estimates); these last are 'predictions' from the logistic regression in the sense of Lane and Nelder [16]. Occurrence was particularly high in the Tel Aviv study of non-alcoholic fatty liver disease. (M37022, 3600586 and the Helsinki study had only one measurement per patient, so do not appear in this analysis, though they did in the analyses of individual abnormal measurements, which are not shown here for brevity.)

There is a slightly higher average occurrence of liver function abnormalities for the orlistat patients: $7.4 \%$ compared to $6.9 \%$ (odds ratio 1.09 or 1.10 from the two analyses, $\mathrm{p}=$ 0.27 or 0.32 ; so the difference between treatments is not statistically significant). This difference is partly accounted for by a larger number of measurements in orlistat patients, due to higher withdrawal rates for placebo patients: 3.60 pairs of successive observations per patient on average, compared to 3.44 on placebo (5\% more). However, it is difficult to adjust for this bias satisfactorily without resorting to an individual-patient analysis that accounts for within-patient correlation of measurements. One approximate approach is to introduce an 'offset variable', as used in the analysis of case-control studies [17] and in Poisson regression to analyze counts associated with varying exposure to risk. This takes 


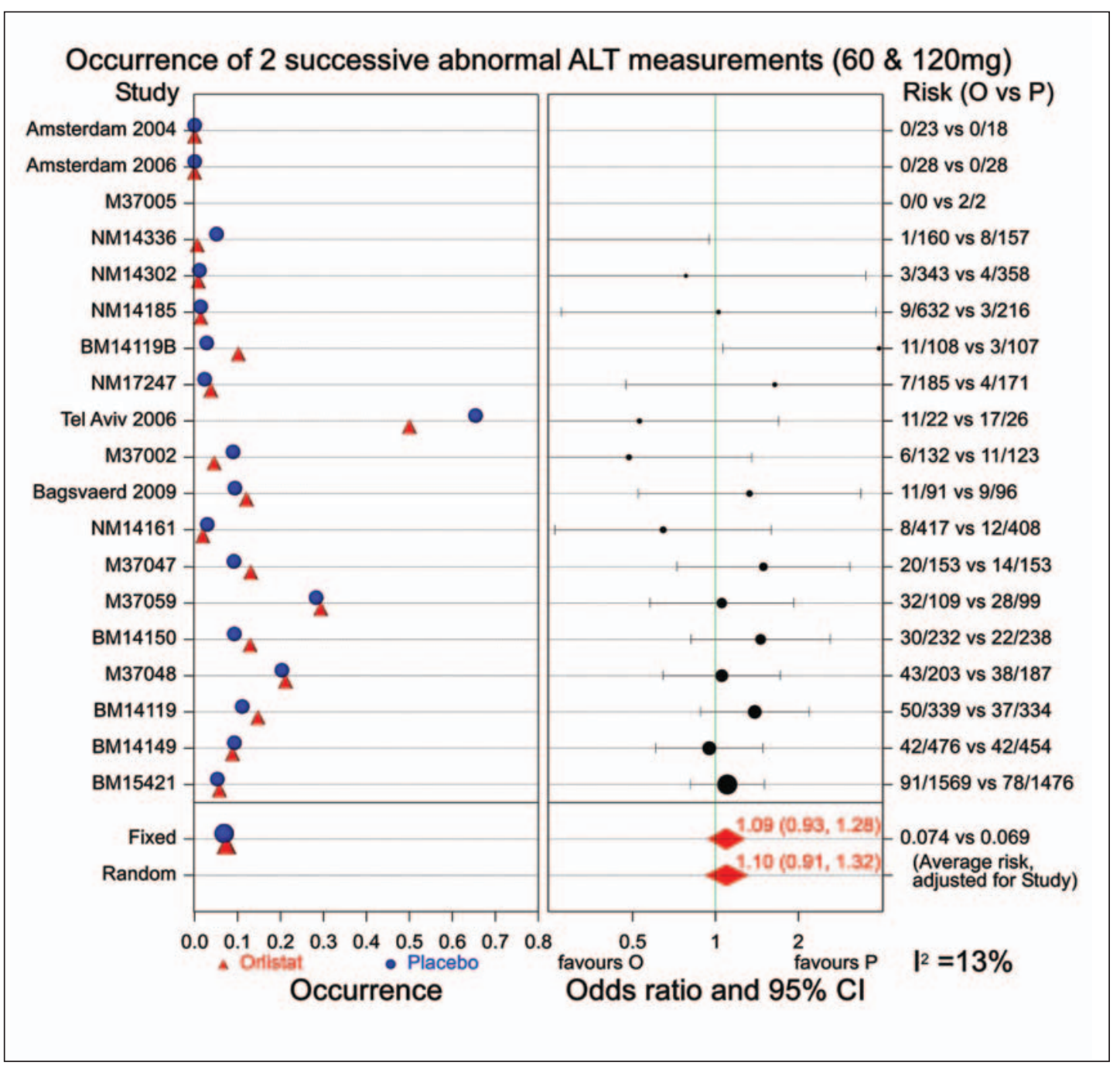

Fig. 1. Occurrence of two successive abnormal ALT measurements (60 and $120 \mathrm{mg}$ orlistat combined).

the form of a covariate in the logistic regression, with no associated parameter, whose value is the logarithm of the mean number of observations. This allows for the different numbers of observations, but takes no account of the likely correlation between values observed on any given patient. The fixed-effect analysis gives an odds ratio of 1.06. A second approach is to analyze the number of patients in each treatment arm who have two successive abnormal values as a proportion of the number of potential pairs of successive observations in that arm. This allows for the different number of observations in each arm, but compromises the assumption of a binomial distribution (needed in logistic regression) because the number of patients with abnormal values cannot be larger than the total number of patients. The fixedeffect analysis gives an odds ratio of 1.05. A third approach would be to analyze the total incidence of two successive abnormal values, allowing more than one per patient, but this is equivalent to the first approach. Because of these drawbacks of summary-level metaanalysis for patients with different exposure, the patient-level approach in the next section should give more reliable results; however, we continue here with the planned analysis because the differences are insubstantial. 


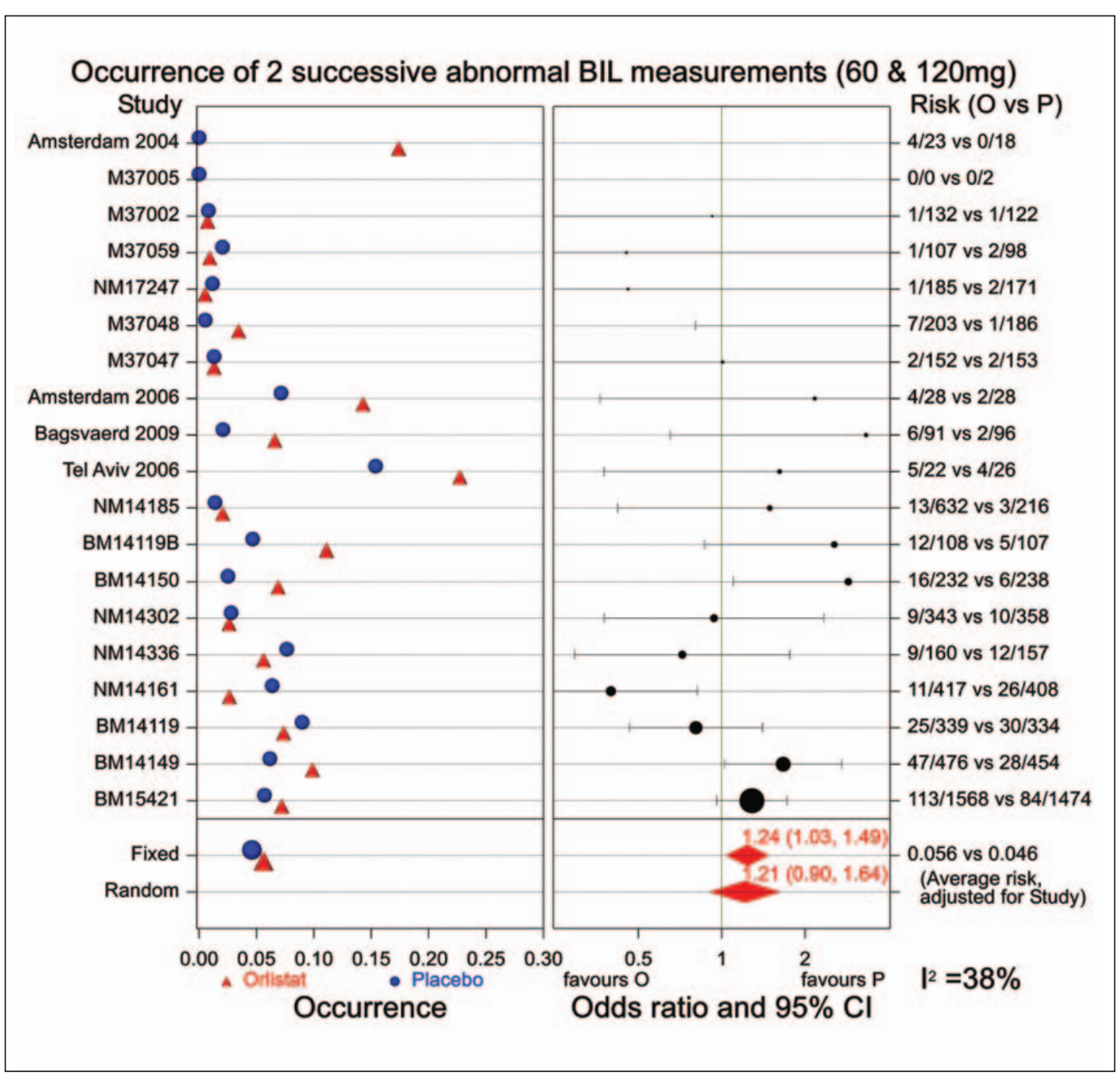

Fig. 2. Occurrence of two successive abnormal bilirubin measurements (60 and $120 \mathrm{mg}$ orlistat combined).

The analysis of individual abnormal measurements was similar to that of two successive abnormal measurements, with odds ratios of 1.05 and 1.04, accounted for by the fact that there were $9 \%$ more measurements on orlistat than on placebo patients. Analyses for $60 \mathrm{mg}$ and $120 \mathrm{mg}$ doses separately gave similar results.

Figure 2 shows the same information for bilirubin. There is more heterogeneity here $\left(\mathrm{I}^{2}=38 \%\right)$, with the two largest studies favoring placebo and the next three largest favoring orlistat, casting doubt on the consistency of the estimates, and hence the usefulness of a fixed-effect estimate [18]. The average occurrence is $5.6 \%$ for orlistat compared to $4.6 \%$ for placebo, with odds ratio 1.24 from the fixed-effect analysis $(p=0.02)$ and 1.21 from the random-effects analysis $(p=0.21)$. Again, this is partly accounted for by the $5 \%$ higher number of observations on orlistat. The analysis of individual abnormal measurements gave average occurrence of $11 \%$ for orlistat compared to $10 \%$, an odds ratio of 1.13 , which was on the boundary of significance at $5 \%$ for both methods of analysis $\left(\mathrm{I}^{2}=0 \%\right)$. But again, there were $9 \%$ more observations for orlistat than for placebo, which accounts for most of the apparent effect. 
Fig. 3. mDISH plot between orlistat (60 and $120 \mathrm{mg}$ combined) and placebo.
Fig. 4. A subject showing high ALT and bilirubin.
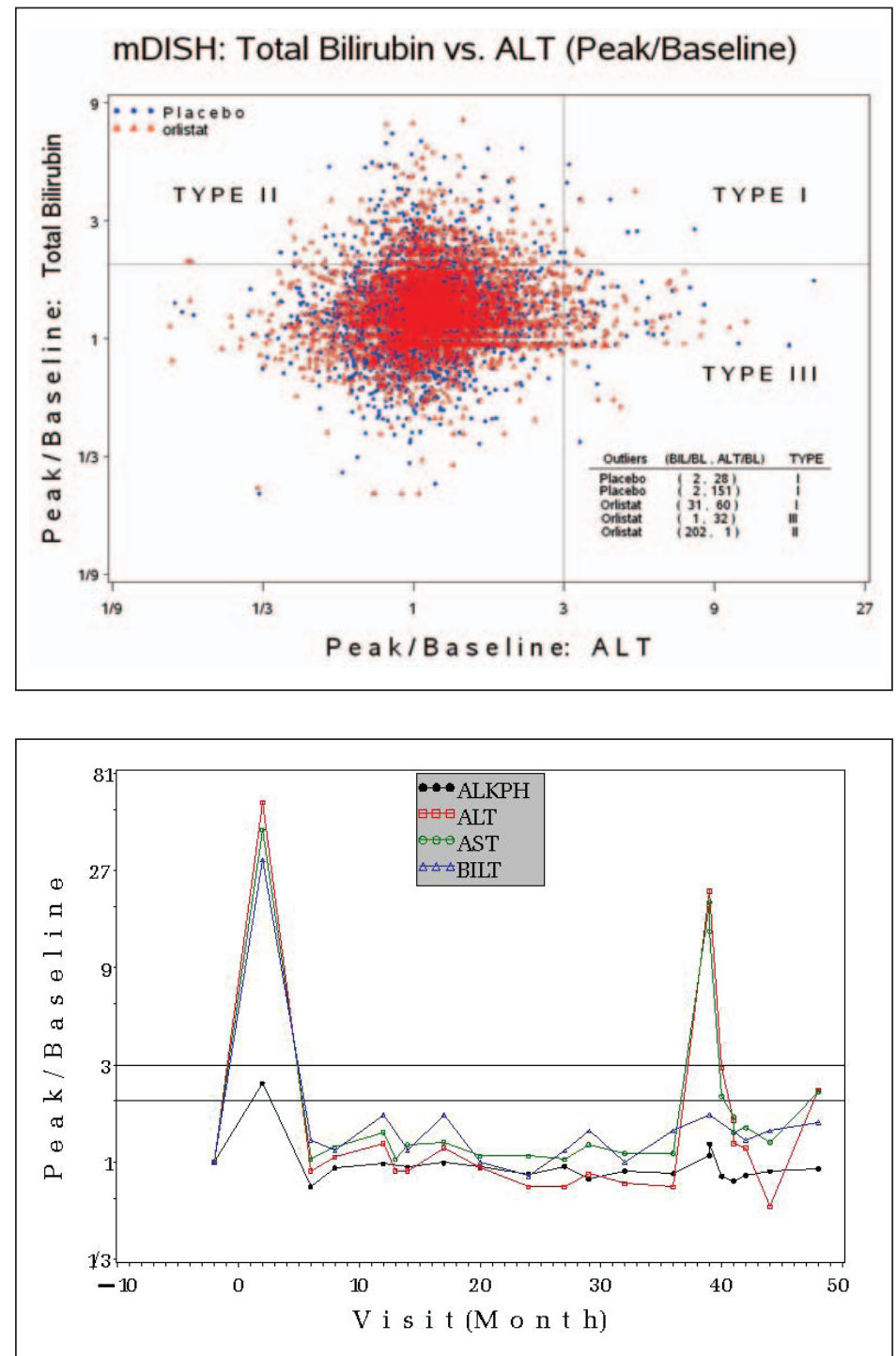

Patient-Level Analysis of ALT and Bilirubin Measurements (mDISH)

In the previous section, we analyzed ALT and bilirubin separately for DILI. In this section, we analyze ALT and bilirubin simultaneously. Figure 3 shows a mDISH plot comparing orlistat treatment (60 and $120 \mathrm{mg}$ combined) to placebo, adjusted for 'regression towards the mean'. The 5 subjects who have larger than $9 \times$ BL of peak bilirubin or $27 \times$ BL of peak ALT are summarized in the outliers table in figure 3. For the Type I subjects (abnormally high ALT and bilirubin), there is a higher frequency of events under placebo $(10 / 4,301=$ $0.0023)$ than under orlistat $(7 / 5,559=0.0013)$. Furthermore, Type I events in patients on placebo show higher elevations of both ALT and bilirubin than those on orlistat. Of interest was a patient on orlistat (see fig. 4) who showed abnormally high ALT and bilirubin levels, but who did not appear to experience any clinical effects. Instead, the data are more consistent with the use of concomitant medicines or viral infection; the subject initially shows elevated levels, which then stabilize until an elevation in month 40 , followed again by stabilization. For the Type II subjects (abnormally high bilirubin only), both treatments show a high and similar proportion of events: placebo $(322 / 4,301=0.075)$ and orlistat 


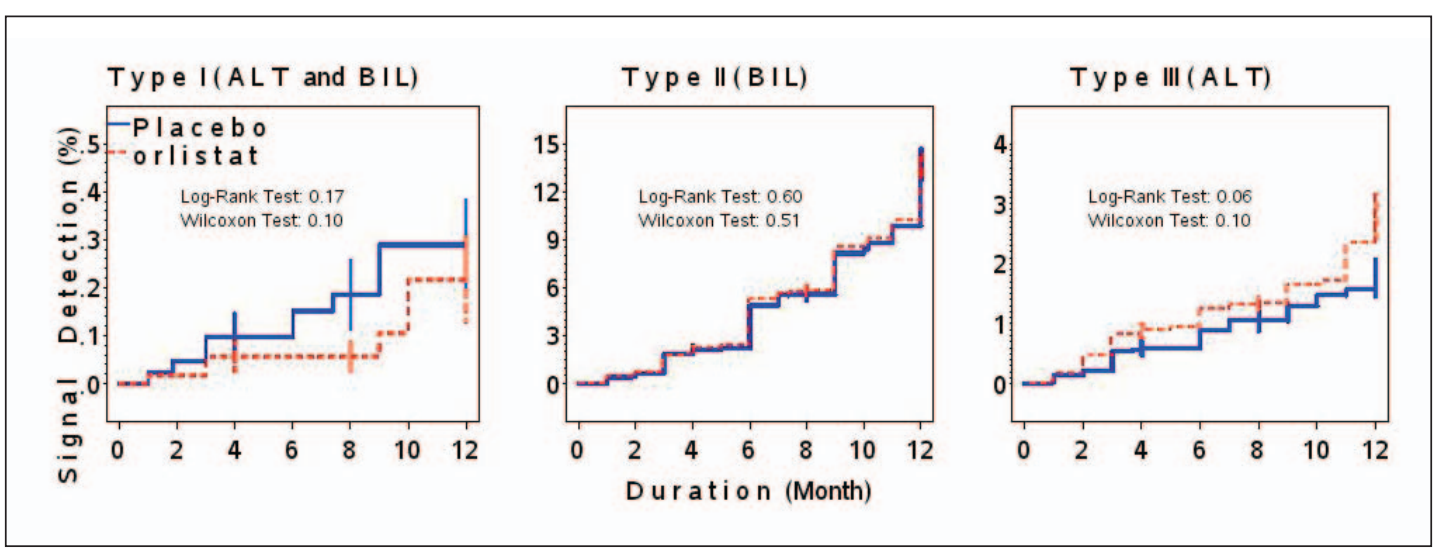

Fig. 5. K-M estimates of difference in treatment-emergent LFT signals between orlistat (60 and $120 \mathrm{mg}$ combined) and placebo. Note: Vertical line represents error bar ( \pm standard error).

$(452 / 5,559=0.081)$. For Type III subjects (abnormally high ALT only), the proportion of events under placebo $(52 / 4,301=0.012)$ was again similar to that of orlistat $(96 / 5,559=$ $0.017)$. Generally, there is no striking distinction between placebo and orlistat based on the frequency rate for Types I, II, and III.

Figure 5 shows results of the K-M analysis. There is little difference between treatments, and none of the differences is statistically significant. For Type II subjects (elevated bilirubin), the treatments look identical ( $p$-value $=0.60$ ). For Type I subjects (both ALT and bilirubin elevated), orlistat is slightly better ( $p$-value $=0.17$ ). For Type III subjects (elevated ALT), placebo is slightly better after 2 months ( $p$-value $=0.06$ ). A few outstanding cases of placebo and orlistat can be regarded as rare events.

\section{Discussion}

Orlistat is an oral gastrointestinal lipase inhibitor and is indicated for treatment of obesity in combination with a hypocaloric diet. There was no evidence of a liver safety signal detected during the clinical trial program; however, post-marketing reports of adverse reactions revealed hints for possible DILI. While a causal relationship has not been established, this has prompted changes to the product information. The goal of the studies presented here was to re-analyze all available and relevant clinical trial data using much more sensitive statistical methods to enable detection of subtle deviations in liver function in subjects during clinical trials. These methods are derived from eDISH as used by FDA accompanied by analyses of events or changes in values over time.

For every case of serious idiosyncratic DILI, one might expect to observe many more cases of asymptomatic injury, detectable only by serum liver function testing. In this metaanalysis, there was no evidence of any important adverse hepatic effect of orlistat in a database of over 10,000 clinical trial subjects. To the best of our knowledge, this analysis incorporated all proprietary and publicly available hepatic safety data from randomized trials of orlistat. The strength of this analysis derives from the reduction of possible confounding factors and the high sensitivity of the novel mDISH method used in the patientlevel analysis.

The use of standard summary-level meta-analysis, which we chose for simplicity when planning the analysis, proved awkward because of the difficulty of dealing satisfactorily 
with different lengths of exposure of subjects to treatments. The effect was small here, with differences between 5 and $9 \%$ in average number of LFT observations between the two treatments, but this is a similar magnitude to the estimated treatment effects from the analysis. This problem is likely to be present in many summary-level meta-analyses of laboratory data, and it is important to be aware that differences in exposure are confounded with the effect of interest. Our study has some limitations. We analyzed all data we could find, but there were many other studies of orlistat from which we were unable to access data. It is therefore possible that there was some bias due to the studies with data not available being different from the others in some way. We think this is unlikely, because any excluded studies are likely to involve relatively few subjects. Secondly, we made no attempt to evaluate the quality of individual studies and their individual potential to be biased. There is potential concern about reporting bias in trials with a drug like orlistat, where blinding is obfuscated by gastrointestinal side effects such as rectal discharge. However, by concentrating on objective laboratory measurements rather than on adverse event reports, we expect to have avoided this. Thirdly, we decided to combine information from all studies we found, despite differences in the populations being studied; for example, some studies were on obese diabetic patients, while the majority of studies involved non-diabetic patients with different severities of obesity. There was certainly some heterogeneity in some of the analyses, which may be associated with the different types of study, but we consider it unlikely to influence our conclusions. Finally, we made no attempt to use detailed subject-level information, beyond treatment and exposure, to model the occurrence of abnormal laboratory values. Covariates such as BMI and the taking of concomitant medications may be related to elevated measurements, but we relied on the randomization process of all the trials to average out any such effect. With $90 \%$ of the manufacturer-sponsored trial subjects being White or Caucasian, we do not think that the racial composition of the database affected the results.

In conclusion, this highly sensitive meta-analysis of orlistat in randomized controlled trials shows no signal for deviations in liver function that might indicate a propensity to hepatotoxicity. As with any medicine, this does not exclude the possibility of idiosyncratic DILI in a large exposed population, but it does show that its potential incidence is likely to be very rare and not predictable. Therefore patient-level analysis is a useful, more sensitive and complementary method to standard summary-level analyses of laboratory drug safety data. It provides investigators the opportunity to detect early safety signals. Importantly no such signal was detected in the analysis of approximately 30,000 patients in the orlistat clinical program.

\section{Acknowledgements}

We are grateful to Roche for providing data from their orlistat studies. We also acknowledge the generous cooperation of those who provided data from external studies: Lisbeth Mathus-Vliegen (Academic Medical Centre, University of Amsterdam), Mia Urjansson (Helsinki and Uusimaa District Hospital, Finland), Angela Harper and Helle Hartvig (Novo Nordisk, Denmark), and Shira Zelber-Sagi (Tel Aviv Sourasky Medical Centre, Israel). We thank Suzanne Brass (Discovery Biometrics, GSK) for carrying out the analyses of binary data.

\section{Disclosure Statement}

This work was conducted by employees of GlaxoSmithKline and sponsored by GlaxoSmithKline and F. Hoffman-la Roche. 
Morris et al.: An Integrated Analysis of Liver Safety Data from Orlistat Clinical Trials

\section{References}

1 Temple RJ, Himmel MH: Safety of newly approved drugs: implications for prescribing. JAMA 2002;287: 2273-2275.

2 US Food and Drug Administration: Questions and Answers: Orlistat and Severe Liver Injury. www.fda.gov/ Drugs/DrugSafety/PostmarketDrugSafetyInformationforPatientsandProviders/ucm213040.htm.

3 Zhi J, Mulligan TE, Hauptman JB: Long-term systemic exposure of orlistat a lipase inhibitor and its metabolites in obese people. J Clin Pharmacol 1999;39:41-46.

4 Lee WM, Senior JR: Recognizing drug-induced liver injury: current problems, possible solutions. Toxicol Pathol 2005;33:155-164.

5 Lee WM: Acute liver failure in the united states. Semin Liver Dis 2003;23:217-226.

- 6 Chitturi S, Farrell GC: Etiopathogenesis of non-alcoholic steatohepatitis. Semin Liver Dis 2001;21:27-41.

- 7 El-Serag HB, Everhart JE: Diabetes increases the risk of acute hepatic failure. Gastroenterology 2002;122: 1822-1828.

- 8 Harrison SA, Fecht W, Brunt EM, Neuschwander-Tetri BA: Orlistat for overweight subjects with non-alcoholic steatohepatitis: a randomized prospective trial. Hepatology 2009;49:80-86.

- 9 Shapiro MA, Lewis JH: Causality assessment of drug-induced hepatotoxicity: promises and pitfalls. Clin Liver Dis 2007;11:477-505.

$\checkmark 10$ Sutton AJ, Cooper NJ, Lambert PC, Jones DR, Abrams KR, Sweeting MJ: Meta-analysis of rare and adverse event data. Expert Rev Pharmacoecon Outcomes Res 2002;2:367-379.

$\checkmark 11$ Zimmerman HJ: The spectrum of hepatotoxicity. Perspect Biol Med 1968;12:135-161.

12 Guo T, Gelperin K, Senior J: A tool to help you decide (detect potentially serious liver injury). 2008. www.fda. gov/downloads/Drugs/ScienceResearch/ResearchAreas/ucm076777.pdf.

13 Cai Z, Christianson M, Stahle L, Keisu M: Reexamining transaminase elevation in Phase I clinical trials: the importance of baseline and change from baseline. Eur J Clin Parmacol 2009;65:1025-1035.

14 Chuang-Stein C: The regression fallacy. Drug Inform J 1993;27:1213-1220.

15 Kaplan EL, Meier P: Nonparametric estimation from incomplete observations. JAm StatistAssoc 1958;53: 457-481.

16 Lane PW, Nelder JA: Analysis of covariance and standardization as instances of prediction. Biometrics 1980;38:613-621.

17 Breslow NE, Cain KC: Logistic regression for two-stage case-control data. Biometrika 1998;75:11-20.

18 Borenstein M, Hedges LV, Higgins JPT, Rothstein HR. Introduction to Meta-Analysis. Chichester, John Wiley \& Sons Ltd., 2009. 